\title{
Erratum to: Regional areas and widths of the midsagittal corpus callosum among HIV-infected patients on stable antiretroviral therapies
}

David F. Tate - Mehul Sampat • Jaroslaw Harezlak •

Mark Fiecas • Joseph Hogan • Jeffrey Dewey •

Daniel McCaffrey • Daniel Branson - Troy Russell • Jared Conley • Michael Taylor • Giovanni Schifitto • J. Zhong • Eric S. Daar • Jeffrey Alger • Mark Brown • Elyse Singer • T. Campbell • D. McMahon • Y. Tso • Janetta Matesan • Scott Letendre • S. Paulose • Michelle Gaugh • C. Tripoli - Constantine Yiannoutsos • Erin D. Bigler • Ronald A. Cohen •

Charles R. G. Guttmann • Bradford Navia • for the HIV Neuroimaging Consortium

Published online: 30 July 2011

(C) Journal of NeuroVirology, Inc. 2011

Erratum to: J. Neurovirol. DOI 10.1007/s13365-011-0033-6

The online version of the original article can be found at http://dx.doi. org/10.1007/s13365-011-0033-6.

D. F. Tate $\cdot$ M. Sampat · J. Dewey $\cdot$ D. McCaffrey $\cdot$ D. Branson •

T. Russell $\cdot$ J. Conley $\cdot$ C. R. G. Guttmann

Departments of Radiology and Psychiatry,

Center for Neurological Imaging, The Brigham and Women's

Hospital, Harvard Medical School,

Boston, MA, USA

D. F. Tate

Department of Neurology, Alzheimer's Disease Center,

Boston University Medical Center,

Boston, MA, USA

J. Harezlak $\cdot$ J. Matesan $\cdot$ C. Yiannoutsos Indiana University School of Medicine,

Indianapolis, IN, USA

M. Fiecas $\cdot$ J. Hogan

Department of Biostatistics, Brown University,

Providence, RI, USA
The name of one of the authors is spelled incorrectly. The correct spelling is Giovanni Schifitto.
M. Taylor $\cdot$ S. Letendre

University of California, San Diego,

San Diego, CA, USA

G. Schifitto $\cdot$ J. Zhong $\cdot$ M. Gaugh

University of Rochester Medical Center,

Rochester, NY, USA

\section{E. S. Daar}

University of California Los Angeles/Harbor,

Torrance, CA, USA

J. Alger - E. Singer

University of California Los Angeles,

Los Angeles, CA, USA

M. Brown · T. Campbell

University of Colorado,

Denver, CO, USA 
$\overline{\text { D. McMahon }} \cdot$ C. Tripoli University of Pittsburgh, Pittsburgh, PA, USA

Y. Tso $\cdot$ S. Paulose Stanford University,

Palo Alto, CA, USA

E. D. Bigler

Departments of Psychology and Neuroscience, Brigham Young University,

Provo, UT, USA

\section{R. A. Cohen}

Brown Medical School, The Miriam Hospital,

Providence, RI, USA

\section{B. Navia}

Departments of Neurology and Psychiatry,

Tufts-New England Medical Center,

Boston, MA, USA

D. F. Tate $(\bowtie)$

Department of Neurology, Henry M. Jackson Foundation,

Defense \& Veterans Brain Injury Center,

San Antonio Military Medical Center,

San Antonio, TX 78324, USA

e-mail: dftatephd@mac.com

B. Navia $(\square)$

Department of Public Health, Jaharis Family Center,

for Biomedical Research,

Tufts Medical School,

Boston, MA 02111, USA

e-mail: bradford.navia@tufts.org 